\title{
Impacto das Condições Socioeconômicas no Desenvolvimento Neuromotor de Crianças de 3 a 12 Meses
}

\author{
Impact of Socioeconomic Conditions on the Neuromotor Development \\ of Children of 3 to 12 Months
}

\author{
Júlia Miranda do Nascimento ${ }^{1}$ \\ Juliana Fonseca de Queiroz Marcelino² \\ Bruna Chagas Almeida ${ }^{3}$ \\ Rita de Cássia Mendonça de Miranda ${ }^{4}$ \\ Raquel Costa Albuquerque ${ }^{5}$
}

\section{RESUMO}

Objetivo: Identificar a relação entre as condições socioeconômicas e o desenvolvimento neuromotor de crianças de 3 a 12 meses. Metodologia: Realizou-se um estudo observacional descritivo com crianças de 3 a 12 meses em uma Unidade de Saúde da Família, Recife-PE. Foram avaliadas 17 crianças e suas famílias, por meio do instrumento Harris Infant Neuromotor Test com o público infantil e um questionário socioeconômico, aplicado com os cuidadores. O resultado do instrumento de avaliação foi obtido em escore, classificados em anormal, suspeito, dentro dos limites normais e avançado. A análise dos dados ocorreu por meio de dois testes estatísticos. O primeiro, Kruskall Wallis, verificou o grau de significância das variáveis do questionário e, posteriormente, o resultado foi gerado no teste ANOVA two way, a fim de correlacionar os itens significativos com todos os escores do instrumento de avaliação. Resultados: Os resultados apontaram que as variáveis: escolaridade, renda familiar e quantidade de pessoas na casa influenciavam os escores obtidos pelas crianças, na avaliação do desenvolvimento, seja de maneira positiva ou negativa. Conclusões: O estudo mostrou a relevância de avaliar o desenvolvimento infantil, bem como os fatores socioeconômicos que o influenciam. Isso permite que os profissionais da área, como o terapeuta ocupacional, identifiquem questões sociais que podem interferir no ganho das habilidades infantis pertinentes de cada faixa etária.

\section{DESCRITORES}

Desenvolvimento Infantil. Classe Social. Terapia Ocupacional.

\begin{abstract}
Objective: Identify the relationship between socioeconomic conditions and neuromotor development of children from 3 to 12 months of birth. Methodology: Observational descriptive approach with children of 3 to 12 months in a Family Health Unit, Recife-PE. 17 children and their families were evaluated. The Harris Infant Neuromotor Test instrument was applied with the children and a socioeconomic questionnaire with the parents. Results: The results were given in scores, classified as abnormal, suspect, within normal limits and advanced. The data analysis was performed using two statistical tests. The Kruskal Wallis test verified the degree of significance of the questionnaire variables and, subsequently, the result was generated in the two-way ANOVA test, to correlate the significant items with all the scores of the assessment instrument. Results: The results showed that the variables: education, family income and number of people in the household influenced the scores obtained by the children, in the assessment of development, either in a positive or negative way. Conclusions: The study showed the relevance of assessing child development, as well as the socioeconomic factors that influence it. This allows professionals in the field, such as the occupational therapist, to identify social issues that may interfere with the gain of the relevant child skills of each age group.
\end{abstract}

\section{DESCRIPTORS}

Child Development. Social Class. Ocupacional Therapy.

\footnotetext{
${ }^{1}$ Terapeuta Ocupacional. Residente do Programa Multiprofissional Integrada em Saúde com ênfase em Saúde da Mulher.

${ }^{2}$ Docente do Curso de Terapia Ocupacional - Universidade Federal de Pernambuco. Doutora em Design pela UFPE.

${ }^{3}$ Terapeuta Ocupacional do Centro Conecta, mestranda em Saúde da Comunicação Humana pela Universidade Federal de Pernambuco. ${ }^{4}$ Docente do Mestrado em Meio Ambiente da Universidade CEUMA. Doutora em Biologia de Fungos pela Universidade Federal de Pernambuco.

${ }^{5}$ Docente do Curso de Terapia Ocupacional - Universidade Federal de Pernambuco. Doutora em Saúde Materno Infantil pelo Instituto de Medicina Integral Professor Fernando Figueira - IMIP.
} 
$\mathrm{O}$ desenvolvimento humano é um processo complexo e contínuo, o qual está relacionado a diversos fatores como biológicos e ambientais, atuantes nos cenários psicossocial, cognitivo e motor ${ }^{1-3}$. $\mathrm{Na}$ primeira infância, o desenvolvimento motor ocorre de forma exponencial, promovendo conexões neuronais mais rápidas $\mathrm{e}$, consequentemente, uma aprendizagem mais acelerada ${ }^{4}$. Por outro lado, é um período vulnerável para o desenvolvimento e ganho de habilidades, tendo em vista a formação dos sistemas e a fragilidade fisiológica dos indivíduos ${ }^{4}$. Assim, a parceria entre a maturação neurológica e a oferta de estímulos do ambiente é fundamental para o desenvolvimento motor. Intercorrências em algum desses aspectos podem influenciar diretamente nas habilidades motoras adquiridas ${ }^{5,6}$.

Os fatores biológicos são imprescindíveis na influência do desenvolvimento humano, contudo, existe uma série de outras variáveis, que são levadas em consideração para determinar, de fato, a composição do indivíduo ${ }^{2}$. Para Palma, Guaríglia e Marques ${ }^{10}$ um importante influenciador para os ganhos das habilidades motoras na primeira infância é o contexto ambiental em que a criança está inserida. Assim, as diferenças socioeconômicas interferem de forma positiva ou negativa no desenvolvimento, seja na oferta dos alimentos, da disponibilidade de brinquedos ou na liberdade para explorar o meio ambiente ${ }^{2,10}$.

Como mecanismo de avaliação do desenvolvimento infantil, os instrumentos de avaliação são utilizados para auxiliar a prática profissional, os quais funcionam como triagem, suporte para encaminhamentos, detecção de atrasos e reavaliações ${ }^{11}$. Segundo
Chaves $^{12}$ as escalas avaliativas passam por um mecanismo complexo de planejamento, construção dos itens, avaliação quantitativa e validação, evidenciando assim, um processo longo que requer estudo e tempo de quem realiza. Rodrigues ${ }^{11}$ afirma que a utilização destes instrumentos é uma estratégia importante para avaliar e identificar atrasos quanto à qualidade das habilidades motoras, bem como a escassez do repertório humano quanto ao desenvolvimento motor.

Desta forma, este estudo justifica-se pela relevância social e epidemiológica em compreender a relação entre as condições socioeconômicos e o desenvolvimento neuromotor infantil e tem como objetivo identificar como estes fatores se relacionam.

\section{METODOLOGIA}

Estudo de abordagem observacional transversal, descritiva, realizado no período de março a maio de 2019, com crianças de 3 a 12 meses, assistidas por uma Unidade de Saúde da Família (USF). Tal método trata da investigação de uma suposição por meio da observação dos seus componentes, não interferindo, portanto, na obtenção de resultados ${ }^{13}$.

Foram aplicados o instrumento de avaliação Harris Infant Neuromotor Test (HINT), a fim de avaliar os aspectos do desenvolvimento neuromotor de bebês de 3 a 12 meses e um questionário com os responsáveis, visando obter informações quanto às características socioeconômicas das famílias ${ }^{14}$.

O HINT é um instrumento que foi desenvolvido no Canadá, de fácil aplicação, no qual o repertório de habilidades neuromotoras das crianças são observados nas posturas 
sentado, deitado em prono, em supino e a transição delas. O resultado do produto destas observações é fornecido em escores, os quais podem ser: avançado, dentro dos limites normais, suspeito e anormal. Tal classificação depende da relação entre a idade e a pontuação obtida a partir das características apresentadas. Para sua aplicação, as crianças foram colocadas em um espaço, no qual pudessem se movimentar livremente no chão, coberto com material emborrachado. Silva ${ }^{14}$ validou o HINT na língua portuguesa, por meio de um estudo longitudinal com crianças atendidas em um centro especializado, em Fortaleza - CE. Como o instrumento requer o uso de diferentes estímulos para incentivar as crianças nas trocas posturais e avaliação das suas habilidades, foi utilizado um kit de estimulação confeccionado pelas pesquisadoras que contém placas de estimulação visual, um aro vermelho e outros utensílios (brinquedos), que não apresentam risco às crianças. Todo o material que compõe o kit está direcionado para o desenvolvimento das habilidades das crianças, em cada grupo etário (3 a 6 - 7 a 9 - 10 a 12 meses).

O questionário socioeconômico, elaborado por um dos pesquisadores, permitiu avaliar o perfil socioeconômico das famílias incluídas e foi aplicado com os cuidadores, abordando itens como: a quantidade de membros que moram na residência, o recebimento de benefício de programa social, o local da residência, o tipo de imóvel que reside, as características do imóvel, as características do saneamento da casa, o tipo de cobertura, o tipo de piso, a quantidade de cômodos da casa, a renda familiar e a escolaridade do entrevistado.
Como critério, foram incluídas crianças registradas na puericultura de uma determinada equipe da Unidade de Saúde Campo do Banco, localizada no bairro da Várzea, em Recife-PE. Foram excluídas aquelas que apresentavam deficiência visual e/ou auditiva diagnosticada e registrada em prontuário médico, que impediam a realização dos itens do instrumento que necessitam destes sistemas como mecanismo de avaliação.

Os resultados obtidos nos "scores" foram submetidos à curva de normalidade para verificação do pareamento dos dados. Por se tratar de dados não paramétricos, as médias foram comparadas po meio do teste de Kruskall Wallis. Com a finalidade de correlacionar os dados amostrais, obtidos em score pelo instrumento avaliativo, com as variáveis significativas do questionário socioeconômico foi realizado o teste de correlação de Spearman, considerando um grau de confiabilidade de $95 \%(p<0.05)$. Todos os testes estatísticos foram realizados com o auxílio do software add Prisma versão 8.

Esta pesquisa foi aprovada pelo Comitê de Ética em Pesquisa da Universidade Federal de Pernambuco, sob o parecer de $\mathrm{n}^{\circ}$. 3.144.419. A coleta somente foi iniciada a partir da assinatura dos pais e/ou responsáveis do Termo de Consentimento Livre e Esclarecido (TCLE) e do Termo de Assentimento.

\section{RESULTADOS}

Foram coletadas informações de 17 crianças e familiares assistidos pela USF Campo do Banco. Assim, foi possível fazer uma caracterização geral do desenvolvimento neuromotor das crianças e as condições 
Tabela 1. Caracterização socioeconômica das famílias de crianças de 3 a 12 meses. Recife, PE

\begin{tabular}{|c|c|c|}
\hline Variáveis & $\mathrm{n}$ & $\%$ \\
\hline \multicolumn{3}{|l|}{ Membros da família } \\
\hline 1 a 2 & 01 & 5,8 \\
\hline 3 a 5 & 12 & 70,5 \\
\hline 6 ou mais & 04 & 23,6 \\
\hline \multicolumn{3}{|c|}{ Benefícios em programas sociais } \\
\hline Sim & 03 & 23,5 \\
\hline Não & 14 & 76,4 \\
\hline \multicolumn{3}{|l|}{ Local de residência } \\
\hline Zona urbana & 17 & 100 \\
\hline \multicolumn{3}{|l|}{ Tipo de imóvel } \\
\hline Alugado & 02 & 11,7 \\
\hline Próprio & 15 & 88,2 \\
\hline \multicolumn{3}{|l|}{ Características da casa } \\
\hline Com acabamento & 11 & 64,7 \\
\hline Sem acabamento & 06 & 35,2 \\
\hline \multicolumn{3}{|l|}{ Saneamento básico } \\
\hline Rede de esgoto & 10 & 58,8 \\
\hline Fossa & 15 & 88,2 \\
\hline Banheiro & 17 & 100 \\
\hline Chuveiro & 17 & 100 \\
\hline Água & 17 & 100 \\
\hline Luz & 17 & 100 \\
\hline \multicolumn{3}{|l|}{ Teto } \\
\hline Laje & 07 & 41,1 \\
\hline Telhas & 10 & 58,8 \\
\hline \multicolumn{3}{|l|}{ Piso } \\
\hline Cimento & 07 & 41,1 \\
\hline Taco & 02 & 11,7 \\
\hline Cerâmica & 07 & 41,1 \\
\hline Outros & 01 & 5,8 \\
\hline \multicolumn{3}{|l|}{ Número de cômodos } \\
\hline 01 & 02 & 11,7 \\
\hline 04 & 04 & 23,5 \\
\hline 05 ou mais & 11 & 64,6 \\
\hline \multicolumn{3}{|l|}{ Renda familiar } \\
\hline Até um SM & 04 & 23,5 \\
\hline Entre 1 e $2 \mathrm{SM}$ & 09 & 52,91 \\
\hline Entre 2 e $3 \mathrm{SM}$ & 02 & 11,7 \\
\hline Entre 3 e 4 SM & 01 & 5,8 \\
\hline Não sabe informar & 01 & 5,8 \\
\hline \multicolumn{3}{|l|}{ Grau de escolaridade } \\
\hline EF incompleto & 03 & 17,6 \\
\hline EF completo & 01 & 5,8 \\
\hline EM incompleto & 03 & 17,6 \\
\hline EM completo & 07 & 35 \\
\hline $\begin{array}{l}\text { ES incompleto } \\
\text { ES completo }\end{array}$ & $\begin{array}{l}02 \\
01\end{array}$ & $\begin{array}{l}11,7 \\
5,8\end{array}$ \\
\hline
\end{tabular}

SM - Salário-Mínimo; EF - Ensino fundamental; EM - Ensino médio; ES - Ensino superior 
Tabela 2. Correlação entre as variáveis dependentes e independentes do estudo. Recife, PE

\begin{tabular}{|c|c|c|c|}
\hline Variável dependente & $\begin{array}{l}\text { Variável independente } \\
\text { (score) }\end{array}$ & P-valor & Diferença \\
\hline 2 & $\begin{array}{c}\text { Dentro dos limites } \\
\text { normais }\end{array}$ & $<0.001$ & -41.00 \\
\hline 3 & $\begin{array}{c}\text { Dentro dos limites } \\
\text { normais }\end{array}$ & $<0.001$ & -42.00 \\
\hline 5 & Suspeito & $<0.05$ & +24.00 \\
\hline 6 & $\begin{array}{c}\text { Dentro dos limites } \\
\text { normais }\end{array}$ & $<0.001$ & -36.00 \\
\hline Mais de 6 & $\begin{array}{c}\text { Dentro dos limites } \\
\text { normais }\end{array}$ & $<0.001$ & -36.00 \\
\hline Até 1 salário mínimo & $\begin{array}{c}\text { Dentro dos limites } \\
\text { normais }\end{array}$ & $<0.05$ & -24.00 \\
\hline $\begin{array}{l}\text { Entre } 1 \text { e } 2 \text { salários } \\
\text { mínimos }\end{array}$ & $\begin{array}{c}\text { Suspeito } \\
\text { Anormal } \\
\text { Avançado }\end{array}$ & $\begin{array}{l}<0.001 \\
<0.1 \\
<0.05\end{array}$ & $\begin{array}{l}+41.00 \\
+35.21 \\
+28.50\end{array}$ \\
\hline $\begin{array}{l}\text { Entre } 3 \text { e } 4 \text { salários } \\
\text { mínimos }\end{array}$ & $\begin{array}{c}\text { Dentro dos limites } \\
\text { normais }\end{array}$ & $<0.001$ & -42.00 \\
\hline $\begin{array}{l}\text { Acima de } 4 \text { salários } \\
\text { mínimos }\end{array}$ & $\begin{array}{c}\text { Dentro dos limites } \\
\text { normais }\end{array}$ & $<0.001$ & -47.00 \\
\hline Não alfabetizado & $\begin{array}{c}\text { Dentro dos limites } \\
\text { normais } \\
\text { Avançado }\end{array}$ & $\begin{array}{l}<0.001 \\
<0.05\end{array}$ & $\begin{array}{l}-47.00 \\
-23.50\end{array}$ \\
\hline $\begin{array}{l}\text { Ensino fundamental } \\
\text { incompleto }\end{array}$ & $\begin{array}{c}\text { Dentro dos limites } \\
\text { normais }\end{array}$ & $<0.1$ & -30.00 \\
\hline $\begin{array}{l}\text { Ensino fundamental } \\
\text { completo }\end{array}$ & $\begin{array}{c}\text { Dentro dos limites } \\
\text { normais }\end{array}$ & $<0.001$ & -42.00 \\
\hline $\begin{array}{l}\text { Ensino médio incom- } \\
\text { pleto }\end{array}$ & $\begin{array}{c}\text { Dentro dos limites } \\
\text { normais }\end{array}$ & $<0.1$ & -30.00 \\
\hline Ensino médio completo & Suspeito & $<0.05$ & +24.00 \\
\hline $\begin{array}{l}\text { Ensino superior incom- } \\
\text { pleto }\end{array}$ & $\begin{array}{c}\text { Dentro dos limites } \\
\text { normais }\end{array}$ & $<0.001$ & -36.00 \\
\hline $\begin{array}{l}\text { Ensino superior com- } \\
\text { pleto }\end{array}$ & $\begin{array}{c}\text { Dentro dos limites } \\
\text { normais }\end{array}$ & $<0.001$ & -42.00 \\
\hline
\end{tabular}

socioeconômicas das famílias, por meio de frequência simples.

A Tabela 1 demonstra a caracterização geral das respostas do questionário socioeconômico. Foi observado que $35,2 \%$ das famílias apresentaram 5 membros morando na mesma casa; $23,5 \%$ recebiam benefícios de progra- mas sociais (bolsa família), $100 \%$ residiam na zona urbana; $88,2 \%$ das famílias tinham imóvel próprio; $100 \%$ apresentavam banheiro, chuveiro, água e luz e, destas, $88,2 \%$ tinham fossa e $58,8 \%$ rede de esgoto; $58,8 \%$ das famílias tinham o teto de telha e $41,1 \%$ tinham piso de cimento; $35,2 \%$ famílias possuíam 
cinco cômodos na casa; $52,71 \%$ famílias receberam entre um e dois salários mínimos e $41,1 \%$ dos cuidadores responsáveis possuíam o ensino médio completo.

O instrumento Harris Infant Neuromotor Test fornece os resultados por meio de escores denominados avançado, dentro dos limites normais, suspeito e anormal. Ele apontou que $8(47 \%)$ crianças avaliadas estavam com o desenvolvimento dentro do esperado para a idade, seguindo de $4(23,5 \%)$ crianças com score avançado, $3(17,6 \%)$ anormal e 2 $(11,7 \%)$ suspeito.

Ocorreu diferença estatisticamente significativa para as variáveis quantidade de membros na casa, escolaridade do cuidador e renda familiar. Foi realizado o cruzamento destas com cada escore e realizada a análise de variância.

Os resultados apontaram que a quantidade de dois e três membros na família influenciou $(p<0,001)$ negativamente nas crianças que estão dentro do esperado para o desenvolvimento neuromotor. Ao correlacionar com quatro membros, não foi identificado nenhuma relação entre esses itens $(p>0,05)$, do mesmo modo que famílias com cinco membros influenciaram fortemente $(p<0,05)$ no desenvolvimento da criança com escore suspeito. Por outro lado, famílias com seis ou mais membros interferiram $(p<0,001)$ negativamente no escore dentro dos limites normais. Os outros escores também foram analisados, porém, não apresentaram relação estatística significativa.

De acordo com os escores obtidos na avaliação infantil e a renda familiar, os resultados da correlação realizada entre esses itens apontaram a influência $(p<0,05)$ negativa com as famílias que recebiam até um salário mínimo. Quando a família tinha a renda mensal entre um e dois salários mínimos passou a ter significativa influência positiva nos escores avançado $(p<0,05)$, anormal $(p<0,1)$ e suspeito $(p<0,01)$, com grau de significância crescente nesta respectiva ordem. Contudo, quando a renda familiar foi a partir de 3 SM passou a influenciar $(p<0.001)$ negativamente no escore dentro dos limites normais. Portanto, esses resultados apontaram que a renda familiar teve influência direta no desenvolvimento neuromotor infantil, indicando que, a partir de $3 \mathrm{SM}$, influenciava negativamente crianças na categoria de escore dentro dos limites normais.

Segundo a relação entre os escores da criança e a escolaridade do cuidador, a correlação apontou que aqueles que não eram alfabetizados influenciaram negativamente as crianças que estavam situadas no escore dentro dos limites normais $(p<0,001) e$ avançadas $(p<0,05)$. Cuidadores com ensino fundamental incompleto $(p<0,1)$ e completo $(p<0,001)$, ensino médio incompleto $(p<0,1)$, ensino superior incompleto $(p<0,001)$ e completo $(p<0,001)$ também influenciaram negativamente nos escores dentro dos limites normais. Em contrapartida, cuidadores com ensino médio completo apresentaram interferência $(p<0,05)$ positiva no grupo de crianças com escore suspeito. Isso mostrou que a escolaridade do cuidador teve relação com o desenvolvimento da criança, de modo que quanto maior a escolaridade torna-se mais propício a incidência do escore dentro dos limites normais. Também se observou uma relação direta da escolaridade com a renda familiar, sendo visível que a medida 
que esses dois itens crescem influenciaram de forma negativa na categoria de escore dentro dos limites normais.

\section{DISCUSSÃO}

A criança, no seu primeiro ano de vida, passa por uma série de transformações que requer uma atenção especial dos profissionais que a acompanha. Portanto, fazem-se necessárias avaliações detalhadas e periódicas, desde o nascimento, com o objetivo de acompanhar e identificar possíveis demandas que possam interferir neste processo. Para isso, os profissionais podem fazer uso de instrumentos de avaliações padronizados, que já apresentam indicadores com parâmetros, avaliando os fatores que englobam o indivíduo ${ }^{16}$. Um dos ambientes que propõem o acompanhamento e vigilância do desenvolvimento infantil é a USF, formada por uma equipe multidisciplinar ${ }^{17}$. Um dos profissionais que a compõe é o terapeuta ocupacional, que, neste contexto, tem o objetivo de avaliar os marcos do desenvolvimento, bem como identificar e propor medidas de estimulação para determinada criança e intervir, inclusive orientar os cuidadores responsáveis ${ }^{17,18}$.

Segundo o Ministério da Saúde do Brasil $^{19}$, as USF são consideradas o primeiro contato da população com a Atenção Básica. Elas são compostas por uma equipe multiprofissional, apta a prestar atenção integral àquele território de sua responsabilidade, além de perceber a necessidade de encaminhamentos para serviços mais especializados. Um dos dispositivos assistenciais ao acompanhamento do desenvolvimento infantil das Equipes de Saúde da Família é a puericultura, que tem a proposta de acompanhar o crescimento das crianças nos seus primeiros meses de vida, fornecendo orientação às famílias quanto as práticas que podem ser beneficiadoras neste processo ${ }^{19,20}$. Por isso, com o intuito de ter um contato direto com um grupo populacional específico, as avaliações do público deste estudo foram realizadas por meio da articulação com uma USF em um serviço que atende crianças de 3 a 12 meses.

Segundo Pereira et al. ${ }^{21}$ e Matos et al. ${ }^{22}$, o desenvolvimento infantil é um processo complexo influenciado por fatores biológicos, como idade gestacional, peso ao nascer e estado nutricional da criança, agregado aos fatores ambientais, como a renda familiar, a ausência da figura paterna, a presença de outras crianças e a escolaridade dos cuidadores. Os indicadores socioeconômicos, então, influenciam de modo geral na forma como a criança irá se desenvolver e adquirir suas habilidades, podendo ter um impacto positivo ou negativo neste processo ${ }^{16,23-25}$. Neste estudo, os resultados apontam a relação que se estabelece entre as condições socioeconômicas da família e o desenvolvimento da criança, principalmente quanto à renda familiar, a escolaridade dos cuidadores e a quantidade de membros que residem na família.

O contexto domiciliar, segundo Zago et al. ${ }^{26}$, é um importante fator ambiental influenciador no desenvolvimento da criança, pois é neste contexto que o indivíduo passa seus primeiros anos, considerado um período de intensa maturação neurológica e ganho de habilidades. A partir da análise dos estudos que apontam a relação entre as condições socioeconômicas e o desenvolvimento infantil, percebe-se um destaque para a renda familiar. 
Este pode ser um dos fatores que influenciam positiva ou negativamente nos eventos neurofisiológicos que acontecem nos primeiros anos de vida e continuam a interferir com o passar do tempo na vida do indivíduo ${ }^{16,24,27}$.

Nesta perspectiva, o presente estudo reafirma que estes fatores se correlacionam entre si, ou seja, responsáveis com alto grau de escolaridade apresentam a tendência a ter uma renda mais alta e, consequentemente, influenciar no desenvolvimento do menor. Contudo, estes resultados apontaram uma influência negativa, inferindo que, quanto maior a escolaridade dos membros das famílias, maior é a renda e o aumento dessas duas variáveis implica na incidência de um escore maior (negativo) para o desenvolvimento. Pode-se pressupor que os familiares que têm escolaridade mais alta e, consequentemente, uma renda maior, passam mais tempo no trabalho, ficando ausentes no ambiente domiciliar e participam menos do processo de estimulação das crianças na fase de desenvolvimento, quando comparado aos cuidadores que conseguem passar um tempo maior em casa.

Segundo Pizzo et al. ${ }^{24}$, a renda familiar pode repercutir diretamente na oferta de brinquedos que a criança é apresentada e, assim, oportunizar meios para o desenvolvimento, visto que o brincar favorece o processo de aprendizagem e permite o desenvolvimento infantil ${ }^{15}$. Em contrapartida, Pizzo et al. ${ }^{24}$ inferem que existem outros meios para a estimulação, por meio do contato com outras pessoas e o incentivo corporal, excluindo, assim, a presença do brinquedo. Compreender os tipos de brincadeiras e brinquedos utilizados pelas crianças é fundamental para avaliação das ha- bilidades que elas apresentam e isso situa os profissionais das áreas de saúde e educação quanto ao que pode se esperar de determinado contexto e a identificar o que pode ser estimulado no infante, para que ele adquira as características neuromotoras pertinentes à sua faixa etária ${ }^{27}$.

Macanae e Comin $^{28}$ afirmam que a baixa renda numa família pode ser o motivo de brigas entre os provedores, influenciando na dinâmica e na relação familiar, repercutindo na oferta de estímulo às crianças, bem como a boa condição socioeconômica da família pode prover meios para um desenvolvimento saudável e tranquilo, refletindo nos ganhos das habilidades do público infantil. Neste estudo, tanto as famílias que têm uma renda abaixo de um salário-mínimo se relacionam de forma negativa, prejudicando o desenvolvimento, como aquelas que têm uma renda acima de 2 salários mínimos. Pressupõe-se, com esse resultado, que familiares com a renda abaixo de um salário mínimo pode indicar a falta de recursos para os cuidados da criança e, assim, dificuldades em oportunizar meios para o desenvolvimento, enquanto, uma renda alta pode indicar a permanência da família no trabalho por um período prolongado, influenciando na estimulação da criança em casa.

A partir dos resultados obtidos, outros aspectos socioeconômicos fazem relação com o desenvolvimento, dentre eles estão à escolaridade dos pais e a quantidade de familiares que residem na mesma casa. Quanto à escolaridade, os achados literários apontam que o nível de instrução dos cuidadores reflete nos cuidados necessários para o crescimento saudável e o entendimento da importância de estimular as crianças quanto aos ganhos das 
habilidades ${ }^{21,25}$. Em concordância, este estudo apontou a influência negativa quando à baixa escolaridade dos pais no desenvolvimento. Contudo, também apontou que escolaridade mais alta (ensino superior) também influenciava negativamente, indo de encontro com outros estudo ${ }^{22}$. Pressupõe-se que, da mesma forma que a renda alta permite que os pais fiquem mais tempo fora de casa, a escolaridade também, deixando, assim, de estimular a criança no ambiente domiciliar.

Não foi encontrado na literatura estudos que relacionem a quantidade de membros na casa com o desenvolvimento infantil, porém, nesta pesquisa, este número foi uma variável que se mostrou influenciadora neste processo. Os resultados apontaram que tanto poucas pessoas na casa (até duas), como muitas pessoas (mais de cinco) vão influenciar de modo negativo na criança, enquanto uma quantidade média influencia de modo a estimular o desenvolvimento.

Pontua-se como limitação da pesquisa a quantidade de crianças e famílias estudadas e as condições de avaliação. Apesar da amostra reduzida, pode-se obter grau significativo do escore do instrumento de avaliação com três variáveis do questionário socioeconômico. Contudo, com uma quantidade maior, possivelmente seria identificar outras correlações com mais fatores socioeconômicos.
Diante do exposto, foi possível identificar os fatores que influenciam no desenvolvimento, bem como se estes são positivos ou negativos para o desenvolvimento neuromotor infantil da população estudada, demonstrando possíveis fatores de risco que predispõem atrasos no desenvolvimento. Isso permite tornar público os dados obtidos, promovendo a construção e efetivação de ações do território e mitigando os fatores que apresentam influência negativa.

\section{CONCLUSÕES}

Por fim, é importante que os profissionais, cuidadores e todos aqueles que lidam com o desenvolvimento infantil entendam quais são os influenciadores neste período e que estejam atentos ao contexto familiar, principalmente no primeiro ano de vida. Isto permite ações voltadas para estimular as crianças, bem como promover intervenções diretas àquelas que foram identificadas com alguma alteração no seu desenvolvimento. Por isso, conhecer esses indicadores torna a prática profissional mais rica e abrangente, permitindo prevenir atrasos maiores no futuro, que podem ser revertidos enquanto a criança é menor se for detectado e estimulado em tempo oportuno.

\section{REFERÊNCIAS}

1- Zago JTDC, Pinto PAF, Leite HR, Santos JN, \& Morais, RLDS. (2017). Association between neuropsychomotor development and biological and environmental risk factors in early childhood children. 320-329. Revista CEFAC. 2017; 19(3).

2- Haywood KM, Getchell M. Desenvolvimento motor ao longo da vida. 6 ed. Porto Alegre: Artmed; 2016.

3- Mello EQD, Motta-Gallo, S, Goulart F C, Herrero, D., \& Gallo PR. Motor development of Brazilian breastfeeding infants in socially unfavorable condition of life, 163-167. Journal of Human Growth and Development. 2014; 24(2)

4- $\quad$ Eickmann SH, Malkes NFDA., \& Lima, MDC, 299-306. Psychomotor development of preterm infants aged 6 to 12 months. São Paulo medical journal, 2012 130(5). 
5- $\quad$ Cypel S. Fundamentos do desenvolvimento Infantil. Brasil: São Paulo; 2011.

6- Clark J. From the Beginning: A Developmental Perspective on Movement and Mobility, 37-45. Nacional Association for Kinesiology and Physical Education in Higher Education, 2005; 57.

7- Tella P, Piccolo LDR, Rangel ML, Rohde LA, Polanczyk GV, Miguel, EC, ... \& Ferraro, AA. (2018). Socio economic diversities and infant development at 6 to 9 months in a poverty area of São Paulo, Brazil. 232-240. Trends in psychiatry and psychotherapy. 2018; 40(3).

8- Formiga CKMR, Vieira MEB., \& Linhares MBM. Developmental assessment of infants born preterm: comparison between the chronological and corrected ages., 230-236. Journal of Human Growth and Development. 2015; 25(2).

9- Silva AJ, Neves LAT, Frônio JS, Ribeiro LC. Factors related developmental delay of newborns, 320-327. Journal of Human Growth and Development. 2014; 24(3).

10- PALMA L, Guaríglia, DA., \& Marques I. (2016). Análise do desenvolvimento motor de crianças em diferentes classes sociais. Coleção de Pesquisa em Educação Física. 2016; 15(2).

11- Rodrigues, OMPR. Employment of scales to evaluate babies' development Educar em Revista. 2012; (43).

12- dos Santos Chaves GDF, de Oliveira AM, Forlenza, O. V., \& Nunes, P. V. Escalas de avaliação para Terapia Ocupacional no Brasil, 240-246 Revista de Terapia Ocupacional da Universidade de São Paulo. 2010; 21(3).

13- Prodanov CC, \& de Freitas EC (2013). Metodologia do trabalho científico: métodos e técnicas da pesquisa e do trabalho acadêmico-2ª Edição. Editora Feevale; 2013.

14- Silva AJ, Neves LAT, FRÔNIO JS, RIBEIRO LC. Factors related developmental delay of newborns, 320-327. Journal of Human Growth and Development. 2014; 24(3).

15- Scalha, TB, Souza VG, Boffi T, \& Carvalho AC. A importância do brincar no desenvolvimento psicomotor: relato de experiência, 79-92. Revista de Psicologia da UNESP. 2017; 9(2)

16- Teixeira MCTV, Alckmin-Carvalho F, Emerich DR, Cevallos PV, \& Paula, CSD. Indicadores de atraso no desenvolvimento em crianças de creche advindas de famílias de baixa renda. 1042-1062. Estudos e Pesquisas em Psicologia. 2017; 17(3).

17- Della Barba PCDS, de Melo Barros V, Figueiredo, MO, \& Martinez LBA (2016). Training in surveillance of child development in the primary health care. Revista Brasileira de Iniciação Científica, 2016; 4(2)

18- de Souza Della Barba PC, de Melo Barros V, de Aquino Marques É, Farias AZ, Aniceto B, \& Miyamoto EE. Occupational Therapy in a process of training on child development's surveillance in primary care health. Cadernos de Terapia Ocupacional da UFSCar, 2017, 25(1).
19- Brasil - Ministério da Saúde. Implantação da Unidade de Saúde da Família. Secretaria de Políticas de Saúde, Cadernos de Atenção Básica, 44, 2000.

20- Barboza CL, Barreto MS, Marcon SS. Records of child care in primary care: descriptive study, 359-375. Online Brazilian Journal of nursing. 2012; 11(3).

21- Pereira JF, Formiga CKMR, Vieira MEB, \& Linhares, MBM Influência dos fatores biológicos e socioeconômicos no desenvolvimento neuropsicomotor de pré-escolares, 135144. Saúde e Pesquisa. 2017; 10(1).

22- Matos LA., Cavalcante LIC, \& Costa, EF. Características do ambiente sociofamiliar e desenvolvimento neuropsicomotor de crianças: associações e implicações, 97108. Revista Subjetividades. 2017; 16(3).

23- Nobre FSS, Bandeira PFR., \& Valentini, NC (2016). Atrasos motores em crianças desfavorecidas socioeconomicamente: Um olhar Bioecológico, 59-69. Motricidade, 2016; 12(2).

24- Pizzo GC, Contreira AR, da Rocha FF, do Nascimento Junior JRA, \& Vieira, LF. Análise das affordances do ambiente domiciliar de crianças pré-escolares: um estudo em função da renda familiar, 79-89. Caderno de Educação Física e Esporte. 2015; 13(1).

25- Zago JTDC, Pinto PAF, Leite HR, Santos JN, \& Morais, RLDS. (2017). Association between neuropsychomotor development and biological and environmental risk factors in early childhood children. 320-329. Revista CEFAC 2017; 19(3).

26- de Souza Morais RL, Carvalho AM, de Castro Magalhães L, \& Pinto, PAF (2016). Primeira infância e pobreza no Brasil: uma análise integrada a partir de indicadores em saúde, educação e desenvolvimento social, 303-314. Revista de Políticas Públicas. 2016; 19(1)

27- de Campos SDF, de Oliveira Figueiredo M, Mazer-Gonçalves, SM, dos Santos, E, \& Maronesi, LC (2017). O brincar para o desenvolvimento do esquema corporal, orientação espacial e temporal: análise de uma intervenção. Cadernos de Terapia Ocupacional da UFSCar, 2017,25(2):275

28- Macanae EC, \& Comim, F. Avaliação do desenvolvimento infantil e a influência da família: uma análise a partir do modelo de equações estruturais MIMIC. Ano do XLIII Encontro Nac Econ, 1-20, 2016.

\section{CORRESPONDÊNCIA}

Júlia Miranda do Nascimento

Endereço: Avenida Conselheiro Aguiar, 855, apt 402. -Recife-PE

E-mail: julia.minas@hotmail.com 